\title{
ORDERING DESIGN TASKS BASED ON COUPLING STRENGTHS
}

\author{
J. L. Rogers \\ NASA Langley Research Center \\ Hampton, Virginia 23681 \\ C. L. Bloebaum \\ Department of Mechanical and Aerospace Engineering \\ State University of New York at Buffalo \\ Buffalo, New York 14260
}

AIAA Paper No. 94-4326

to be presented at the

Fifth AIAA/NASA/USAF/ISSMO Symposium on Multidisciplinary Analysis and Optimization

Panama City, Florida

September 7-9, 1994 


\title{
ORDERING DESIGN TASKS BASED ON COUPLING STRENGTHS
}

\author{
J. L. Rogers* \\ NASA Langley Research Center \\ C. L. Bloebaum** \\ Department of Mechanical and Aerospace Engineering \\ State University of New York at Buffalo
}

\begin{abstract}
$\underline{\text { Abstract }}$
The design process associated with large engineering systems requires an initial decomposition of the complex system into modules of design tasks which are coupled through the transference of output data. In analyzing or optimizing such a coupled system, it is essential to be able to determine which interactions figure prominently enough to significantly affect the accuracy of the system solution. Many decomposition approaches assume the capability is available to determine what design tasks and interactions exist and what order of execution will be imposed during the analysis process. Unfortunately, this is often a complex problem and beyond the capabilities of a human design manager. A new feature for DeMAID (Design Manager's Aid for Intelligent Decomposition) will allow the design manager to use coupling strength information to find a proper sequence for ordering the design tasks. In addition, these coupling strengths aid in deciding if certain tasks or couplings could be removed (or temporarily suspended) from consideration to achieve computational savings without a significant loss of system accuracy. New rules are presented and two small test cases are used to show the effects of using coupling strengths in this manner.
\end{abstract}

\section{$\underline{\text { Introduction }}$}

Many design projects are large and multidisciplinary in nature. Before the design of complex systems can begin, a significant amount of time and money must be invested in determining the order of and the interactions among the design tasks. This is particularly true if each task is performed by a separate group of engineers or if the multidisciplinary analysis is to be incorporated

\footnotetext{
* Senior Computer Scientist.

** Assistant Professor, Member AIAA.

Copyright @1994 by the American Institute of Aeronautics and

Astronautics, Inc. No copyright is asserted in the United States under Title 17, U.S. Code. The U.S. Government has a royalty-free license to exercise all rights under the copyright claimed herein for Governmental purposes. All other rights are reserved by the Copyright Owner.
}

within an optimization procedure. In 1989, a new knowledge-based tool [1] was developed to aid the design manager in ordering these tasks, determining their interactions, grouping iterative processes, and displaying the entire process in the format of a design structure matrix $(\mathrm{NxN})$. This tool is called the Design Manager's Aid for Intelligent Decomposition (DeMAID). Since its release to the public, new enhancements have been incorporated into DeMAID [2]. Now that DeMAID has been distributed to several industries and universities, feedback from surveys indicate that DeMAID users are not only interested in just ordering the design process, but want to use DeMAID as an aid in reengineering the design problem around the flow of information and to emphasize the tracking of cost and time.

Much of the cost and time of a design project is incurred in the iterative processes called circuits. These are tight groupings of tasks which must be repeated until the outputs converge to stable values. DeMAID currently groups all the tasks in their respective circuits and orders the tasks within the circuit to minimize the number of feedback loops. This ordering may or may not be the most efficient in terms of cost and time, since several different orderings within a circuit may yield the same number of feedbacks. One method for making the ordering more efficient is to take advantage of the strengths of the couplings (interfaces) among the tasks [3]. This paper gives a brief overview of new directions for DeMAID, followed by a discussion of ordering the design process based on coupling strengths.

\section{New Directions for DeMAID}

When DeMAID was first developed, the knowledge base was written around rules for organizing multidisciplinary design and optimization problems with design variables, behavior variables, constraints, and an objective function. As more people started to use DeMAID, it quickly became apparent that the tool was applicable to a much broader range of problems requiring new capabilities. In addition to just ordering 
the design tasks, determining the interfaces, grouping the tasks into iterative processes called circuits, and decomposing the circuits into a hierarchical display, design managers have a need to see what tasks are affected if a change is made to some data or task in the design process. In addition to tracking the flow of tasks, they want to be able to track the flow of information through the design process. They want to track costs and time in design, manufacturing, and maintenance; and use DeMAID to aid in determining where simplifications might be made.

To aid in determining the effects of a change in the design process, new rules for selecting tasks for reexecution were added to DeMAID [2]. As an example, Figure 1 displays a portion of a design structure matrix. In this figure, the boxes represent modules, a horizontal line from a box represents an output produced by that module, and a vertical line to a box represents an input required by that module. A dot indicates a coupling between two modules because the output of one module (e.g. module 25) provides input to other modules (e.g. modules 27 and 28). If a change is made to the input of module 5, a design manager may want to know what effects it would have on the output of module 29. Would all modules between module 5 and 29 have to be re-executed or only a portion?

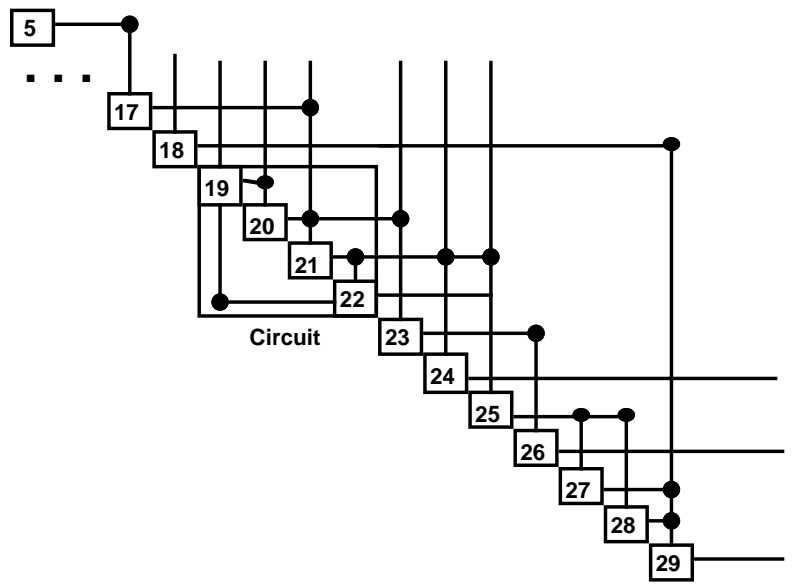

Figure 1. Design structure matrix.

Figure 2 shows that only a portion of the modules (those that are not shaded) require re-execution. Although the output from module 18 is an input to module 29 , this output is not affected by a change to module 5. Modules 23, 24, and 26 may be affected by the changes to module 5 , but since their output does not change any input to module 29 , they too may be omitted from re-execution. Since one or more of these tasks may require substantial resources in cost, time, personnel, and/or equipment, any task not having to be re-executed may result in a significant savings.

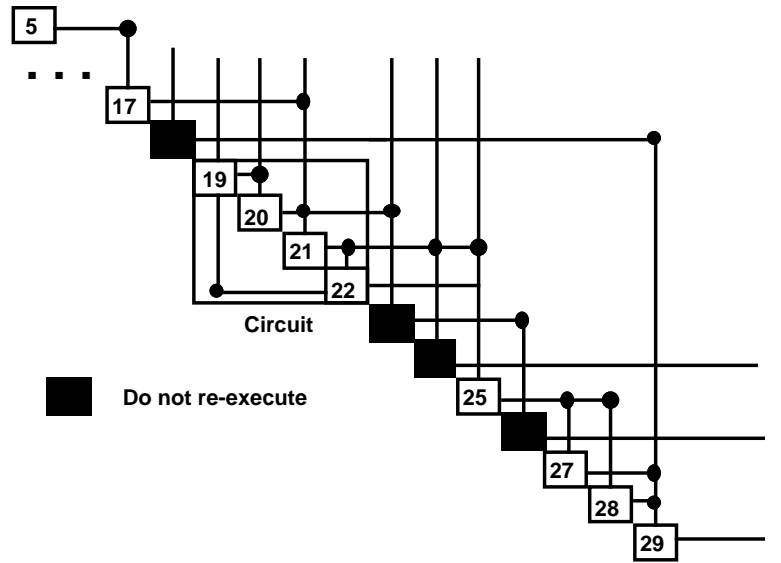

Figure 2. Modified design structure matrix.

To enable the design manager to track more detailed information requires a change in the way the output is represented in the format of a module definition. Initially [1], the format was:

\section{(module number name type time output status input-list)}

where the output field signifies all output produced by a module, whether it be a single piece of data, a string of data, a vector, a matrix, or any combination. Design managers have expressed their desire to see more details than the present representation allows.

For example, suppose a module represents a finite element analysis program which computes stresses, displacements, vibration modes, etc. In the original format, all this output would be lumped into a single name (e.g. feout ).

\section{(module 1 m1 125 feout uk fein geom)}

In Figure 3, this task is represented as module 1, and has interfaces to modules 2, 3, and 4. Hence, these modules require feout as input. Suppose however that module 2 really only requires stresses as input to compute stress constraints, module 3 only requires displacements to compute displacement constraints, and module 4 requires stresses, stress constraints, and displacements. The design structure matrix would appear the same, but if a change occurred that only affected the stresses, then module 3 would not have to be re-executed since only the displacement data from feout are used as input. 


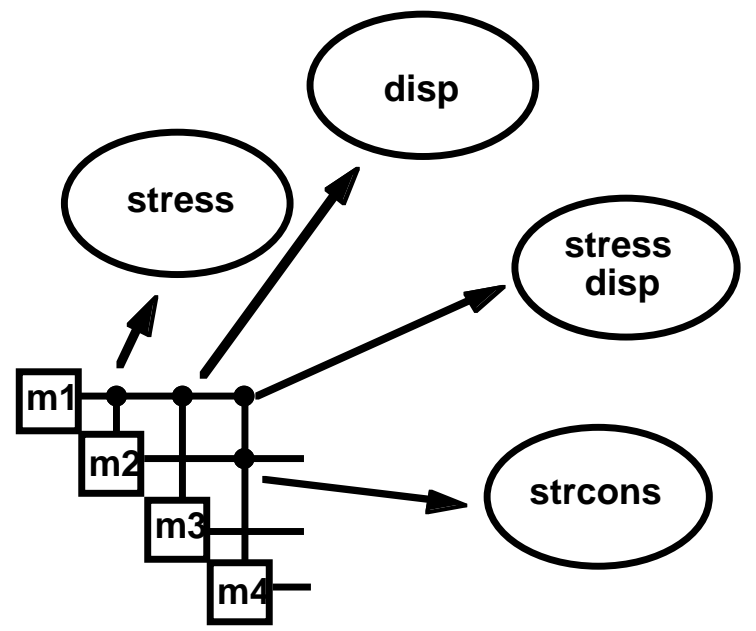

(module $1 \mathrm{~m} 1125$ feout uk)

(module 2 m2 110 strcons uk)

(output feout stress disp)

(requires uk VS strcons stress)

Figure 3. Modules with multiple outputs.

The design manager can now trace specific pieces of data and be as detailed as the problem requires. This is accomplished by adding two new sets of lists (examples in Figure 3) to the DeMAID input. The user creates the module list as before, but omits the input-list. DeMAID uses the two new lists to create the input-list for each module.

The first new list is an output list in the format:

\section{(output name output-breakdown)}

where the name in the output list matches the output in the module list. The output-breakdown in the output list is a breakdown of all the pieces of output data the design manager needs to trace.

The second new list aids in determining the interfaces. It has the format:

\section{(requires status strength output input)}

where the output and input fields are elements of output-breakdown from the output lists and the strength field represents the coupling strength between those two elements.

The concept of coupling strengths becomes very important when trying to reduce costs and cycle time. Since much of the cost and time of a design project are incurred in the iterative processes called circuits, these coupling strengths can aid in deciding if certain tasks or couplings can be removed (or temporarily suspended) from consideration to achieve computational savings without a substantial loss of system accuracy. DeMAID handles seven levels of coupling strengths: (1) extremely weak $(\mathrm{EW}),(2)$ very weak $(\mathrm{VW}),(3)$ weak (W), (4) nominal (N), (5) strong (S), (6) very strong (VS), and (7) extremely strong (ES). Currently, the user must estimate and input these coupling strengths if the requires lists are needed. The sections below describe a method for computing the coupling strengths as well as rules for using them to reduce time and costs with minimal loss in accuracy.

\section{Coupling Strengths}

\section{Coupling Strength Definition}

Here, coupling strengths are defined in terms of the local normalized sensitivities. Since these local sensitivities are used in the Global Sensitivity Equations (GSE) to obtain total behavioral response derivatives, they are already available to the design manager [4].

The GSE approach involves using the chain rule to define the total derivatives of the output response quantities in terms of local sensitivities of each subsystem. The GSE's feasibility has been demonstrated in previous work $[4,5,6]$. Once the local sensitivities are known, the total derivatives of the output response quantities with respect to the design variables can be determined from the solution of the matrix set of global sensitivity equations. Since the $\mathrm{dY} / \mathrm{dX}$ information is invariably required in design for trade-off studies, as well as formal gradient-based optimization, it is inevitably available during the design process.

Since the components of the output response vector Y and the design variable vector $\mathrm{X}$ are of varying magnitudes from one subsystem to another, it is necessary to scale the local sensitivities. Hence, a normalization scheme [7] can be implemented to ensure that the conditioning of the system is such that accuracy of the solution is not threatened. The local normalized sensitivities (denoted by ') for a sample two subsystem (A and B) problem are:

$$
\frac{\partial Y A^{\prime}}{\partial Y B}=\frac{Y B}{Y A} \frac{\partial Y A}{\partial Y B} \quad \text { and } \quad \frac{\partial Y B^{\prime}}{\partial Y A}=\frac{Y A}{Y B} \frac{\partial Y B}{\partial Y A}
$$




\section{$\underline{\text { Sensitivity-Based Heuristics }}$}

The local subsystem sensitivity information can be used to quantify the strengths of participating analysis couplings. This coupling information can then be used to provide the basis for developing heuristics that indicate which couplings are "weak" enough to be temporarily or permanently suspended. For example, if it is determined, from the system in Figure 4a, that the coupling from module 4 to module 3 , and from module 5 to module 3 are negligible (i.e. $\partial Y 3 / \partial Y 4^{\prime}$ and $\partial Y 3 / \partial Y 5^{\prime}$ are extremely small in comparison to other normalized local sensitivities), it may be decided to temporarily suspend the feedback loop among these modules, thereby reducing the complexity of the problem to that seen in Figure $4 b$.
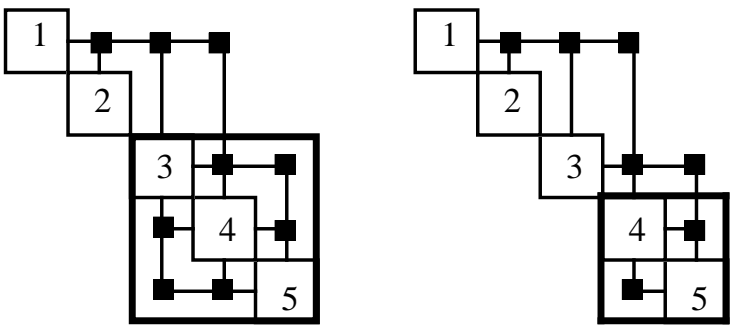

Figures $4 \mathrm{a}$ and $\mathrm{b}$. Subsystem modules with full and modified dependencies.

Obviously, in a complex problem involving computationally expensive analyses (such as structural finite element analyses) that must be executed within an iterative framework, the ability to reduce the system complexity without sacrificing solution accuracy is of the utmost importance.

The question then becomes, "to what extent may solution accuracy be compromised in order to achieve solution efficiency?" It is this question that is addressed in the development of new rules for DeMAID. The application of a knowledge-based system enables the answer to this question to be largely problem dependent, thus increasing applicability and flexibility.

\section{Coupling Strength Quantification}

In this paper, a linear distribution between upper and lower bounds of the local sensitivity space is used to quantify the seven levels of coupling strengths.

One possible approach is to calculate the mean and standard deviation where the mean value of the local normalized sensitivity derivatives $\left(\mathrm{s}_{\mathrm{i}}\right)$ can be determined from

$$
\overline{\mathrm{s}}=\frac{1}{\mathrm{~N}} \sum_{\mathrm{i}=1}^{\mathrm{N}} \mathrm{s}_{\mathrm{i}}
$$

where $\mathrm{N}$ is the number of couplings. The associated standard deviation can now be determined from the relation,

$$
\sigma(\mathrm{s})=\left[\frac{1}{\mathrm{~N}-1} \sum_{\mathrm{i}=1}^{\mathrm{N}}\left(\mathrm{s}_{\mathrm{i}}-\overline{\mathrm{s}}\right)^{2}\right]^{1 / 2}
$$

The upper and lower bounds of the local normalized sensitivity space are defined in terms of the mean value and standard deviation as,

$$
\begin{aligned}
& \mathrm{s}^{\mathrm{u}}=\overline{\mathrm{s}}+k_{1} \sigma(\mathrm{s}) \\
& \mathrm{s}^{1}=\overline{\mathrm{s}}-k_{2} \sigma(\mathrm{s})
\end{aligned}
$$

where $\mathrm{k}_{1}$ and $\mathrm{k}_{2}$ are user-prescribed values based on experience and heuristics. Anything outside these bounds is either extremely weak (EW) or extremely strong (ES).

Once these bounds are defined, associated coupling strengths can be assigned to all other couplings based on a linear distribution between the bounds. Hence,

$$
\begin{aligned}
& \text { if } \quad s_{i} \geq \bar{s}+k_{1} \sigma(s) \quad \text { then } s_{i} \text { is ES } \\
& \text { if } \quad \bar{s}+\frac{3}{5} k_{1} \sigma(s) \leq s_{i}<\bar{s}+k_{1} \sigma(s) \quad \text { then } \quad s_{i} \text { is } V S \\
& \text { if } \bar{s}+\frac{1}{5} k_{1} \sigma(s) \leq s_{i}<\bar{s}+\frac{3}{5} k_{1} \sigma(s) \text { then } s_{i} \text { is } S \\
& \text { if } \bar{s}-\frac{1}{5} k_{2} \sigma(s)<s_{i}<\bar{s}+\frac{1}{5} k_{1} \sigma(s) \text { then } s_{i} \text { is } N \\
& \text { if } \bar{s}-\frac{3}{5} k_{2} \sigma(s)<s_{i} \leq \bar{s}-\frac{1}{5} k_{2} \sigma(s) \text { then } s_{i} \text { is } W \\
& \text { if } \quad \bar{s}-k_{2} \sigma(s)<s_{i} \leq \bar{s}-\frac{3}{5} k_{2} \sigma(s) \quad \text { then } s_{i} \text { is } V W \\
& \text { if } \quad s_{i} \leq \bar{s}-k_{2} \sigma(s) \quad \text { then } s_{i} \text { is } E W
\end{aligned}
$$

Another possible approach to quantifying these coupling strengths is more heuristics-based. Once the mean is determined as above, an upper and lower limit on the normalized sensitivity space can be prescribed by the design manager. The range for the nominal quantification can also be pre-determined, leaving a linear distribution for the quantifications between the upper limit and the nominal range and those between the lower limit and the nominal range. For example, if the upper bound on the normalized sensitivity space is $\mathrm{UB}$, the lower bound is $\mathrm{LB}$, and the distance from the mean to the nominal boundary is NB, the quantifications would be classified as, 


$$
\begin{aligned}
& \text { if } s_{i} \geq U B \\
& \text { then } s_{i} \text { is ES } \\
& \text { if } \quad \frac{(\bar{s}+N B)+U B}{2} \leq s_{i}<U B \\
& \text { then } s_{i} \text { is VS } \\
& \text { if } \bar{s}+N B \leq s_{i}<\frac{(\bar{s}+N B)+U B}{2} \\
& \text { if } \bar{s}-N B<s_{i}<\bar{s}+N B \\
& \text { if } \quad \frac{(\bar{s}-N B)-L B}{2}<s_{i} \leq \bar{s}-N B \\
& \text { if } \quad L B<s_{i} \leq \frac{(\bar{s}-N B)-L B}{2} \\
& \text { if } s_{i} \leq L B \\
& \text { then } s_{i} \text { is } S \\
& \text { then } s_{i} \text { is } N \\
& \text { then } s_{i} \text { is W } \\
& \text { then } s_{i} \text { is } V W \\
& \text { then } s_{i} \text { is EW }
\end{aligned}
$$

The coupling strength quantifications are then supplied to DeMAID in the requires list.

\section{DeMAID Coupling Strength Rules}

Once the coupling strengths have been determined, they can be used with a new set of DeMAID rules to determine which modules may possibly be removed (or temporarily suspended) from the design process. The rules work in the following manner. First, all modules with at least one coupling having a nominal $(\mathrm{N})$ or stronger strength are retained. Modules with only extremely weak (EW) coupling strengths are removed. The remaining modules are checked to determine their relationship to other modules in the system. The maximum coupling strength is found for each module remaining in the system. If a module has a weak (W) maximum coupling strength and interfaces with a module with a very strong or extremely strong (VS or ES) coupling strength, then that module is retained. Otherwise, it is removed from consideration. If a module has a very weak (VW) maximum coupling strength and interfaces with a module with an extremely strong (ES) coupling strength, then that module is retained. Otherwise, it too is removed from consideration. All references to the output generated by the removed modules are deleted from the input lists of the remaining modules. The user can now input this reduced set of modules into DeMAID.

\section{Application to Analytical Systems}

\section{Intelligent Decomposition}

Before describing the application of the new DeMAID rules to analytical systems, it is necessary to understand how coupling strength information would be used in a design procedure. Figure 5 demonstrates how DeMAID, together with the coupling strength data, can significantly reduce the number of both full system analyses as well as full optimization runs. The design manager's judgment would be used to identify all modules believed to be important for the complex system. DeMAID would yield an ordering of those participating tasks based on its planning and scheduling components. A full analysis would then be performed followed by determination of sensitivity information. From the local normalized sensitivities, coupling strengths would be quantified and categorized as extremely weak (EW), very weak (VW), etc. They would then be supplied to DeMAID to determine the reduced system and its associated ordering of tasks. The reduced system and it's optimization problem would be solved for some number of prescribed cycles, n, after which the full system and new coupling strengths would be evaluated. This approach results in the potential for substantially reduced computational times with minimal loss in system and optimization accuracy.

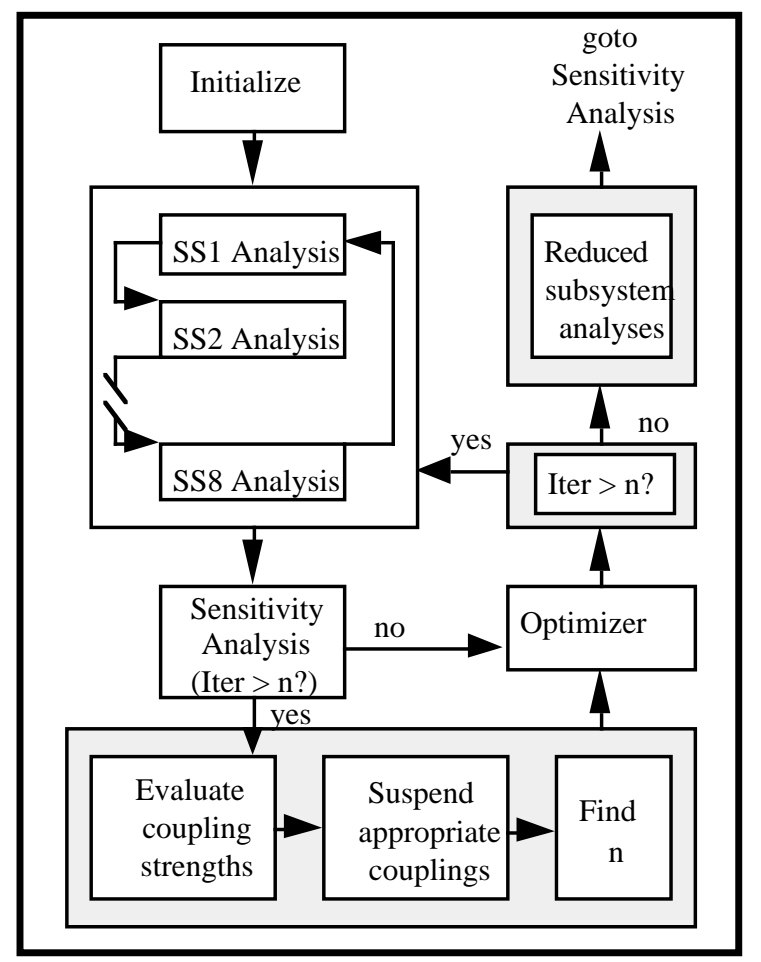

Figure 5. Interactions in two subsystem non-hierarchic environment.

Two analytical problems have been developed to emulate a complex engineering system with lateral couplings. Figure 6 demonstrates the coupling for the first analytical system. Within each subsystem, outputs are associated with contributing functions. For example, within subsystem 1 of Case 1 (Figure 6), there are two functions associated with Subsystem 1, S1W1 with output w1 and S1W2, with output w2. These outputs, which may be individual or multiple pieces of data, then form input to other subsystems, and 
hence, functions within those subsystems. The same is true for the system of Case 2.

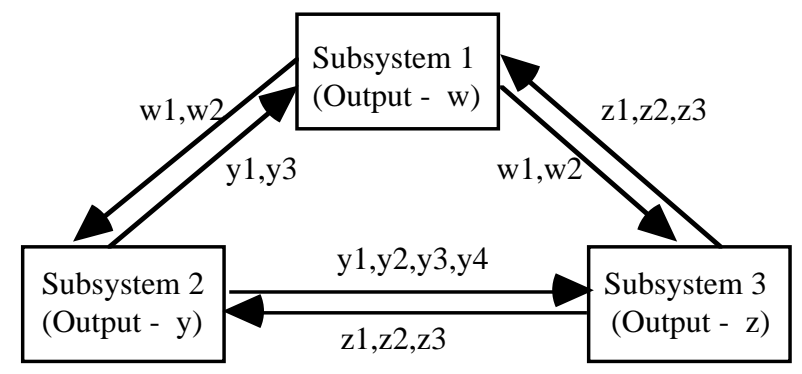

Figure 6. Non-hierarchic analytical system providing testbed for coupling strength comparisons.

\section{Analytical Coupled System Analyses}

Implicit relations are defined for both cases for each subsystem. The notation $\mathrm{f}($. . .) denotes a functional relationship among the listed inputs and outputs. The explicit equations for Case 1 are as defined in [3], while those for Case 2 are listed in the Appendix.

\section{Case 1:}

Subsystem 1 (SS1)

$\mathbf{X} 1=(\mathrm{x} 11, \mathrm{x} 12)$ and

$\mathrm{w} 1=\mathrm{f}(\mathbf{X} 1, \mathrm{y} 3, \mathrm{z} 1, \mathrm{z} 3)$

w2 $=\mathrm{f}(\mathbf{X} 1, \mathrm{y} 1, \mathrm{y} 3, \mathrm{z} 2)$

Subsystem 2(SS2)

$\mathbf{X} 2=(\mathrm{x} 21, \mathrm{x} 22, \mathrm{x} 23)$ and

$\mathrm{y} 1=\mathrm{f}(\mathbf{X} 2, \mathrm{w} 1, \mathrm{w} 2, \mathrm{z} 2)$

$\mathrm{y} 2=\mathrm{f}(\mathbf{X} 2, \mathrm{w} 2, \mathrm{z} 1, \mathrm{z} 2)$

$\mathrm{y} 3=\mathrm{f}(\mathbf{X} 2, \mathrm{z} 1, \mathrm{z} 3)$

$\mathrm{y} 4=\mathrm{f}(\mathbf{X} 2, \mathrm{w} 1, \mathrm{z} 2)$

Subsystem 3 (SS3)

$\mathbf{X} 3=(\mathrm{x} 31, \mathrm{x} 32)$ and

$\mathrm{z} 1=\mathrm{f}(\mathrm{X} 3, \mathrm{w} 2, \mathrm{y} 2, \mathrm{y} 4)$

$\mathrm{z} 2=\mathrm{f}(\mathbf{X} 3, \mathrm{w} 1, \mathrm{y} 1)$

$\mathrm{z} 3=\mathrm{f}(\mathbf{X} 3, \mathrm{y} 3, \mathrm{w} 2, \mathrm{y} 4)$

The application of DeMAID to the above system of coupled functions results in the ordering shown in Figure 7. Each function is denoted by a reference to its subsystem and to the output associated with it. Modules pertaining to the design variables (e.g. S1X1) are included to make the identification of subsystem inputs easier.

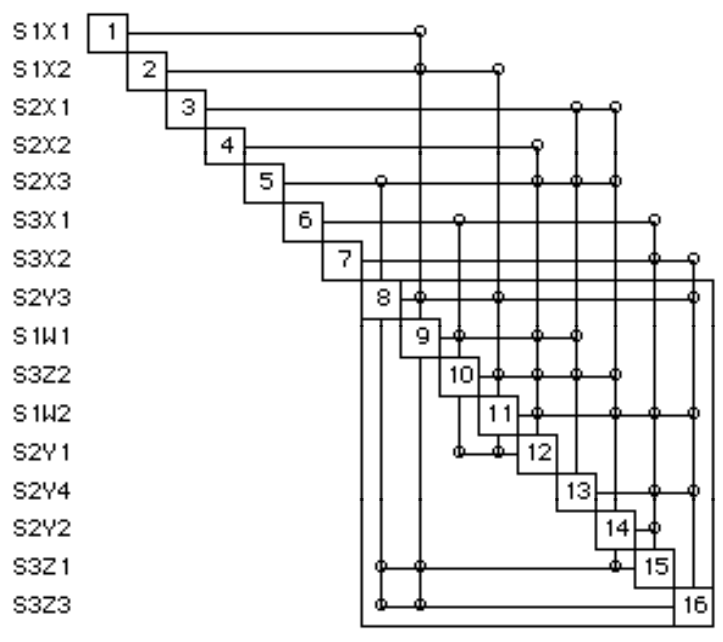

Figure 7. Case 1 modules with minimized feedbacks.

\section{Case 2:}

$\underline{\text { Subsystem 1 (SS1) }}$

$\mathbf{X} 1=(\mathrm{x} 11, \mathrm{x} 12, \mathrm{x} 13)$ and

$\mathrm{t} 1=\mathrm{f}(\mathbf{X} 1, \mathrm{y} 1, \mathrm{v} 2)$

t2 $=\mathrm{f}(\mathbf{X} 1, \mathrm{v} 1, \mathrm{z} 1)$

t3 $=\mathrm{f}(\mathbf{X} 1, \mathrm{v} 2, \mathrm{z} 2, \mathrm{u} 2)$

$\mathrm{t} 4=\mathrm{f}(\mathrm{X} 1, \mathrm{w} 2, \mathrm{v} 4, \mathrm{y} 3)$

Subsystem 2 (SS2)

$\mathrm{X} 2=(\mathrm{x} 21, \mathrm{x} 22)$ and

$\mathrm{u} 1=\mathrm{f}(\mathbf{X} 2, \mathrm{z} 1, \mathrm{w} 3)$

$\mathrm{u} 2=\mathrm{f}(\mathbf{X} 2, \mathrm{t} 1, \mathrm{t} 3, \mathrm{w} 2, \mathrm{z} 3)$

$\mathrm{u} 3=\mathrm{f}(\mathbf{X} 2, \mathrm{t} 1, \mathrm{y} 1)$

Subsystem 3(SS3)

$\mathbf{X} 3=(\mathrm{x} 31, \mathrm{x} 32, \mathrm{x} 33)$ and

$\mathrm{v} 1=\mathrm{f}(\mathbf{X} 3, \mathrm{t} 2, \mathrm{u} 2, \mathrm{z} 1, \mathrm{w} 2)$

$\mathrm{v} 2=\mathrm{f}(\mathbf{X} 3, \mathrm{t} 1, \mathrm{y} 2)$

v3 $=\mathrm{f}(\mathbf{X} 3, \mathrm{u} 3, \mathrm{w} 1)$

$\mathrm{v} 4=\mathrm{f}(\mathbf{X} 3, \mathrm{y} 1, \mathrm{y} 3, \mathrm{t} 4, \mathrm{w} 3, \mathrm{u} 1)$

Subsystem 4 (SS4)

$\mathrm{X} 4=(\mathrm{x} 41, \mathrm{x} 42, \mathrm{x} 43)$ and

$\mathrm{w} 1=\mathrm{f}(\mathbf{X} 4, \mathrm{y} 1, \mathrm{y} 2, \mathrm{z} 2)$

w2 $=\mathrm{f}(\mathbf{X} 4, \mathrm{z} 1, \mathrm{z} 2, \mathrm{z} 3, \mathrm{u} 2)$

w3 $=\mathrm{f}(\mathbf{X} 4, \mathrm{t} 4, \mathrm{v} 4)$

$\underline{\text { Subsystem } 5 \text { (SS5) }}$

$\mathrm{X} 5=(\mathrm{x} 51, \mathrm{x} 52, \mathrm{x} 53)$ and

$\mathrm{y} 1=\mathrm{f}(\mathbf{X} 5, \mathrm{t} 1, \mathrm{u} 3, \mathrm{v} 3)$

y2 $=f(\mathbf{X} 5, \mathrm{u} 3, \mathrm{w} 1, \mathrm{v} 2)$

$\mathrm{y} 3=\mathrm{f}(\mathbf{X} 5, \mathrm{z} 3, \mathrm{t} 4, \mathrm{u} 1)$

$\mathrm{y} 4=\mathrm{f}(\mathbf{X} 5, \mathrm{t} 3, \mathrm{u} 2, \mathrm{z} 3)$

Subsystem 6(SS6)

$\mathrm{X} 6=(\mathrm{x} 61, \mathrm{x} 62)$ and

$\mathrm{z} 1=\mathrm{f}(\mathbf{X} 6, \mathrm{t} 2, \mathrm{t} 3, \mathrm{v} 1)$

$\mathrm{z} 2=\mathrm{f}(\mathbf{X} 6, \mathrm{v} 2, \mathrm{v} 3, \mathrm{w} 1)$

$\mathrm{z} 3=\mathrm{f}(\mathrm{X} 6, \mathrm{w} 1, \mathrm{w} 2, \mathrm{t} 2, \mathrm{u} 2, \mathrm{y} 4)$ 
Figure 8 shows the full system for Case 2, with all couplings.

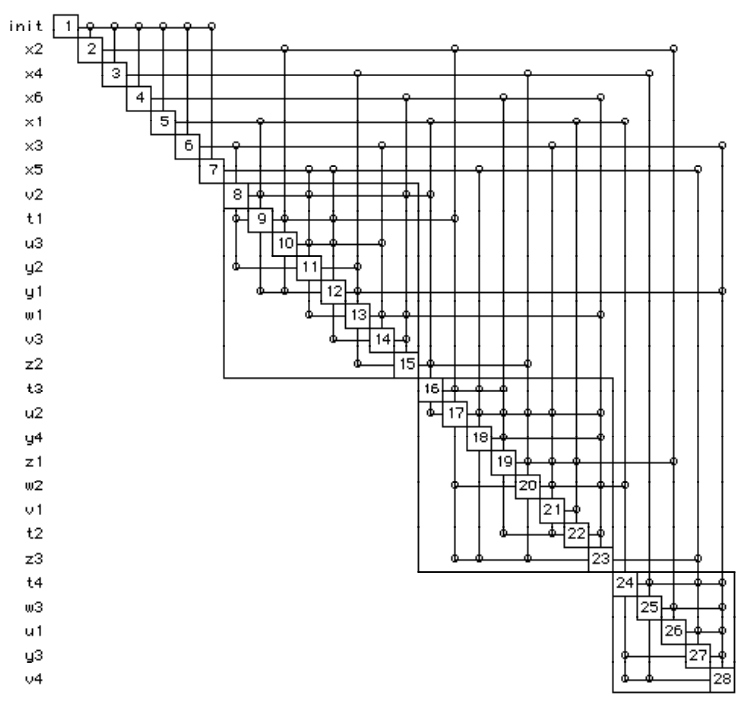

Figure 8. Case 2 modules with minimized feedbacks.

\section{Discussion of Results}

The first step in applying the DeMAID-based reduction scheme is to quantify the coupling strengths for Cases 1 and 2. The results of applying the heuristics-based quantification scheme previously described are shown in Table 1 for Case 1 and Table 2 for Case 2. The distribution for the normalized local sensitivity space is shown in Figures 9 and 10 for the two cases.

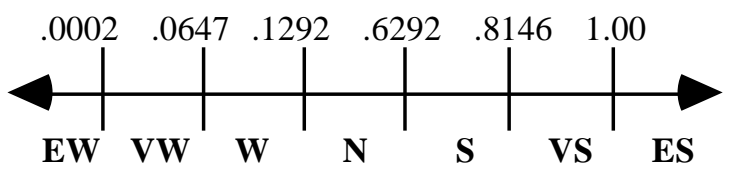

Figure 9. Case 1 boundaries for quantifying coupling strengths.

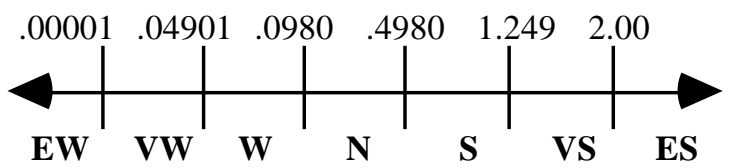

Figure 10. Case 2 boundaries for quantifying coupling strengths.

For Case 1, the new rules resulted in modules S3Z2 and S3Z3 being removed from consideration as in the original paper presenting this case [3]. As reported in that paper, the largest percent difference, $7.153 \%$, was in the solution for w2. The next largest, $2.28 \%$ was associated with $\mathrm{y} 1$, and all others were below $1 \%$.
For Case 2, the same set of rules allowed modules v3, $\mathrm{w} 2, \mathrm{y} 4$, and $\mathrm{z} 3$ to be removed. As can be seen from Table 3 the output values for the reduced system are very close to those of the full system. The removed modules are shaded in the table. Removing the modules, however, produced an interesting change in the design structure matrix as shown in Figure 11. One module was removed from the top circuit, reducing the feedbacks by one. The bottom circuit was unchanged. Three modules were removed from the middle circuit resulting in a division into two smaller circuits.

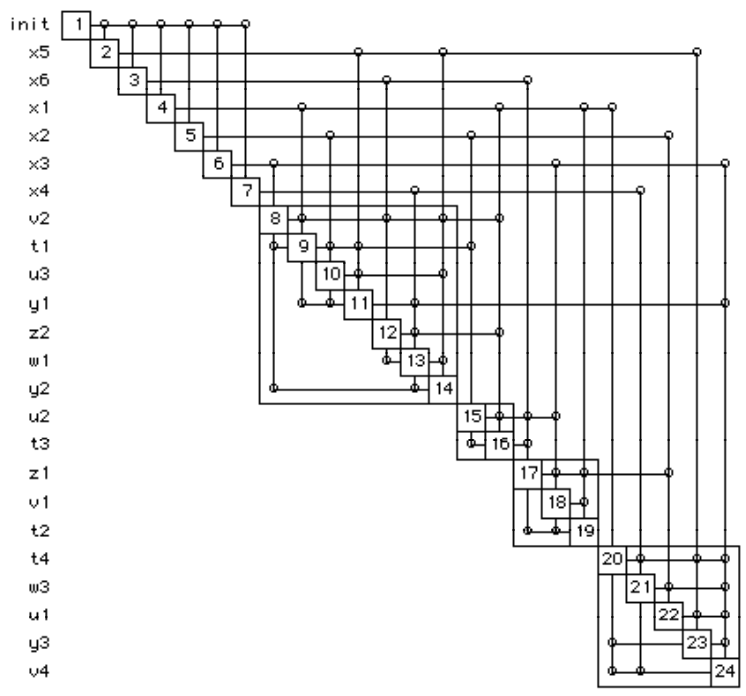

Figure 11. Case 2 after modules removed

With these two cases, it has been shown that general rules can be developed to remove (or temporarily suspend) modules from execution by examining the strengths of the couplings among the modules. The strengths or weaknesses of individual couplings can be determined by applying statistical techniques to sensitivity data. With this knowledge, modules may be removed from the design process which offers a potential improvement in both time and cost without a loss in solution accuracy. 
Table 1. Coupling Strength Quantifications for Case 1 using heuristic approach.

\begin{tabular}{|l|c|c|c|c|c|c|c|c|c|} 
& \multicolumn{1}{c}{ delw1/ } & delw2/ & dely $1 /$ & dely2/ & dely3/ & dely4/ & delz1/ & delz2/ & delz3/ \\
\hline delw1 & & & ES & & & W & & VW & \\
\hline delw2 & & & N & VW & & & VW & & W \\
\hline dely1 & & VW & & & & & & VW & \\
\hline dely2 & & & & & & & VW & & \\
\hline dely3 & VW & VW & & & & & & & VS \\
\hline dely4 & & & & & & & S & & EW \\
\hline delz1 & VW & & & VW & VW & & & & \\
\hline delz2 & & W & VW & EW & & EW & & & \\
\hline delz3 & EW & & & & VW & & & & \\
\hline
\end{tabular}

Table 2. Coupling Strength Quantifications for Case 2 using heuristic approach.

\begin{tabular}{|c|c|c|c|c|c|c|c|c|c|c|c|c|c|c|c|c|c|c|c|c|c|}
\hline$\partial \theta$ & $\mathrm{t} 1 / /$ & 121 & $\mathrm{t} 3 /$ & $\mathrm{t} 4 /$ & $\mathrm{u} 1 /$ & $\mathrm{u} 2 /$ & $\mathrm{u} 31$ & $\mathrm{v1} / /$ & $\mathrm{v} 21$ & $\mathrm{v} 3 /$ & $\mathrm{v} 4 /$ & w1/ & $\mathrm{w} 2 /$ & w3/ & $\mathrm{y} 1 / 1$ & $\mathrm{y} 2 /$ & $\mathrm{y} 3 /$ & $\mathrm{y} 4 /$ & $\mathrm{z} 1 /$ & $\mathrm{z} 21$ & $\mathrm{z} 3 /$ \\
\hline $\mathrm{t1}$ & & & & & & $\mathrm{EW}$ & VW & & $\mathrm{S}$ & & & & & & $\bar{N}$ & & & & & & \\
\hline$\overline{t 2}$ & & & & & & & & $\mathrm{~W}$ & & & & & & & & & & & VW & & VW \\
\hline t3 & & & & & & $\bar{N}$ & & & & & & & & & & & & $\bar{N}$ & VW & & \\
\hline$\overline{t 4}$ & & & & & & & & & & & VW & & & $\mathrm{N}$ & & & VW & & & & \\
\hline $\mathrm{ul}$ & & & & & & & & & & & $\mathrm{VW}$ & & & & & & $\mathrm{VW}$ & & & & \\
\hline u2 & & & $\mathrm{N}$ & & & & & $\mathrm{VW}$ & & & & & $\mathrm{W}$ & & & & & VW & & & VW \\
\hline u3 & & & & & & & & & & $\mathrm{VW}$ & & & & & VW & $\mathrm{W}$ & & & & & 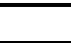 \\
\hline $\mathrm{v} 1$ & & ES & & & & & & & & & & & & & & & & & VW & & \\
\hline $\mathrm{v} 2$ & $\mathrm{~N}$ & & $\mathrm{ES}$ & & & & & & & & & & & & & $\mathrm{S}$ & & & & $\mathrm{N}$ & \\
\hline v3 & & & & & & & & & & & & & & & VW & & & & & EW & \\
\hline $\mathrm{v} 4$ & & & & $\mathrm{ES}$ & & & & & & & & & & $\mathrm{S}$ & & & & & & & \\
\hline w1 & & & & & & & & & & $\mathrm{N}$ & & & & & & $\mathrm{VW}$ & & & & $\mathrm{VW}$ & $\mathrm{N}$ \\
\hline w2 & & & & $\mathrm{VW}$ & & $\mathrm{VW}$ & & $\mathrm{VW}$ & & & & & & & & & & & & & VW \\
\hline w3 & & & & & $\mathrm{W}$ & & & & & & $\overline{\mathrm{VW}}$ & & & & & & & & & & \\
\hline $\mathrm{y} 1$ & $\mathrm{~W}$ & & & & & & VW & & & & $\mathrm{N}$ & $\mathrm{N}$ & & & & & & & & & \\
\hline $\mathrm{y} 2$ & & & & & & & & & $\mathrm{~N}$ & & & VS & & & & & & & & & \\
\hline $\mathrm{y} 3$ & & & & VW & & & & & & & $\mathrm{VW}$ & & & & & & & & & & \\
\hline $\mathrm{y} 4$ & & & & & & & & & & & & & & & & & & & & & VW \\
\hline $\mathrm{z} 1$ & & ES & & & $\mathrm{N}$ & & & $\mathrm{VW}$ & & & & & ES & & & & & & & & \\
\hline $\mathrm{z2}$ & & & $\mathrm{N}$ & & & & & & & & & EW & $\mathrm{N}$ & & & & & & & & \\
\hline $\mathrm{z3}$ & & & & & & $\mathrm{VW}$ & & & & & & & EW & & & & VW & VW & & & \\
\hline
\end{tabular}


Table 3. Comparison of output values for full and reduced systems for Case 2.

\begin{tabular}{|c|c|c|}
\hline Output & Full System & Reduced System \\
\hline t 1 & 71.3021 & 71.3019 \\
\hline t 2 & 31.5566 & 31.5560 \\
\hline t 3 & 94.8302 & 94.8267 \\
\hline t 4 & 38.7446 & 38.7472 \\
\hline u 1 & 70.3609 & 70.3600 \\
\hline u 2 & 90.1629 & 90.1616 \\
\hline u 3 & 37.4936 & 37.4936 \\
\hline v 1 & 135.3255 & 135.3257 \\
\hline v 2 & 95.4775 & 95.4776 \\
\hline v 3 & 143.4225 & 143.4225 \\
\hline v 4 & 119.3493 & 119.3497 \\
\hline w 1 & 141.7335 & 141.7328 \\
\hline w 2 & 34.4332 & 34.4332 \\
\hline w 3 & 132.2914 & 132.2894 \\
\hline y 1 & 139.2781 & 139.2783 \\
\hline y 2 & 81.6617 & 81.6616 \\
\hline y 3 & 147.3626 & 147.3626 \\
\hline y 4 & 95.5950 & 95.5950 \\
\hline z 1 & 141.3610 & 141.3611 \\
\hline z 2 & 77.4906 & 77.4906 \\
\hline z 3 & 110.3259 & 110.3259 \\
\hline
\end{tabular}

\section{Concluding Remarks}

Much of the cost and time of a design project are incurred in the iterative processes called circuits. A new method for making the ordering within the circuits more efficient by taking advantage of the strengths of the couplings among the tasks was introduced. This new method applies new rules in the knowledge base based on coupling strength information derived from sensitivity data. These rules determine which modules may be removed (or temporarily suspended) from the design process without sacrificing solution accuracy. Two coupled systems composed of analytical equations were tested for verification purposes using the same rules. In both cases, modules were removed based on the sensitivity data with little or no effect on the solution.

\section{Acknowledgments}

The second author would like to acknowledge partial support of this work under NSF RIA grant \#DDM 9309706.

\section{$\underline{\text { References }}$}

1. Rogers, J.L., "A Knowledge-Based Tool for Multilevel Decomposition of a Complex Design Problem", NASA TP 2903, May 1989.

2. Rogers, J.L. and Barthelemy, J.-F. M., "Enhancements to the Design Manager's Aid for Intelligent Decomposition (DeMAID)", AIAA Paper No. 92-4809, 1992.

3. Bloebaum, C.L., "An Intelligent Decomposition Approach for Coupled Engineering Systems", AIAA Paper No. 92-4821, 1992.

4. Sobieszczanski-Sobieski, J., "On the Sensitivity of Complex, Internally Coupled Systems", AIAA Journal, Volume 28, No. 2, 1990, pp. 153-160.

5. Sobieszczanski-Sobieski, J., Bloebaum, C.L., and Hajela, P., "Sensitivity of Control-Augmented Structure Obtained by a System Decomposition Method", AIAA Journal,. Volume 29, No. 2, 1991, pp. 264-270.

6. Bloebaum, C.L., "Global Sensitivity Analysis in Control-Augmented Structural Synthesis", AIAA Paper No. 89-0844, 1989.

7. Hajela P., Bloebaum, C.L., and SobieszczanskiSobieski, J., "Application of Global Sensitivity Equations in Multidisciplinary Aircraft Synthesis", Journal of Aircraft, Volume 27, No. 12, 1990, pp. 1002-1010. 


\section{Appendix}

Explicit analytical expressions for each subsystem analysis of Case 2 are presented. Each output quantity (e.g. w1) is assumed to represent output from a subroutine. Table A1 shows coefficient values used in the system analysis.

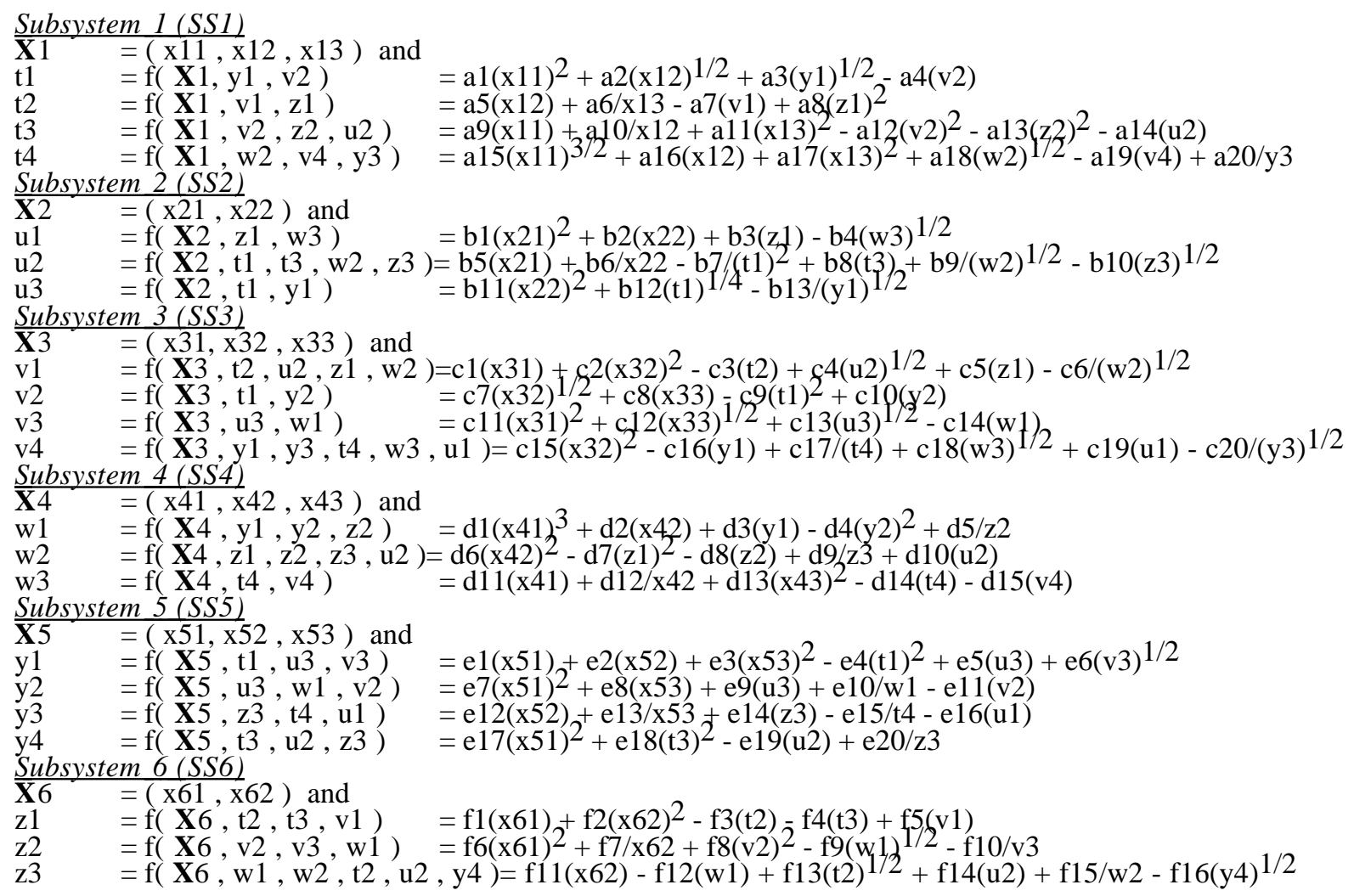

Table A1. Coefficient Values for Analytical System of Case 2.

\begin{tabular}{|c|c|}
\hline Coefficients & Coefficient values \\
\hline a1-a20 & 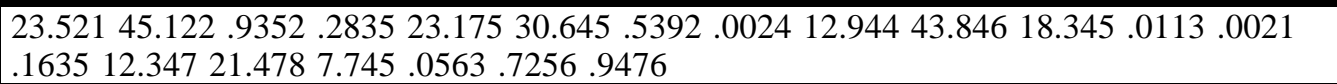 \\
\hline b1-b13 & 8.43512 .563 .1577 .938824 .54724 .3561 .457 .3846 .9862 .73779 .367 .0285 .6758 \\
\hline c1-c20 & 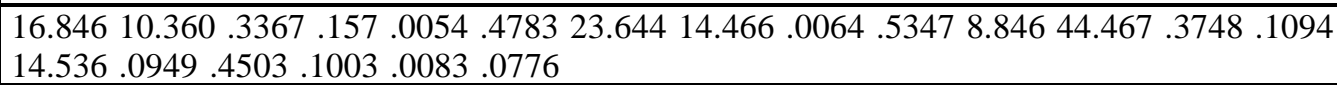 \\
\hline d1-d15 & $\begin{array}{l}14.89613 .746 .4567 .0144 .094416 .436 .0113 .0673 .0356 .02758 .63826 .6936 .536 .6685 \\
.8467\end{array}$ \\
\hline e1-e20 & $\begin{array}{l}27.783 \quad 35.55210 .377 .0059 .0983 .063315 .36718 .653 .1079 .2763 .83122 .56135 .649 \\
.6463 .7366 .06679 .882 .0011 .0359 .2033 \\
\end{array}$ \\
\hline f1-f16 & $\begin{array}{l}8.6297 .932 .0274 .0235 .002114 .36748 .837 .0009 .0328 .082529 .663 .0846 .0024 .0436 \\
.0875 .0289\end{array}$ \\
\hline
\end{tabular}

